\title{
Synthesis of pyrazolo[4,3-c] $[1,2,6]$ benzothiadiazocine, a new ring system as potential COX inhibitor
}

\author{
Onofrio Migliara, Virginia Spanò, Barbara Parrino, Cristina Ciancimino, \\ and Patrizia Diana*
}

Dipartimento di Scienze e Tecnologie Molecolari e Biomolecolari (STEMBIO), Università di Palermo, Via Archirafi 32, 90123 Palermo, Italy

E-mail: patrizia.diana@unipa.it

\section{Dedicated to Prof. Anthony J. Arduengo on the occasion of his $60^{\text {th }}$ anniversary}

\begin{abstract}
Derivatives of the new ring system 1,4-dihydropyrazolo[4,3-c][1,2,6] benzothiadiazocin$11(10 H)$ one 5,5-dioxide were synthesized in five or six steps in 57-66\% overall yields and tested as COX inhibitors.
\end{abstract}

Keywords: $\quad[1,2,6]$ Benzothiadiazocine, 1,4-dihydropyrazolo[4,3-c][1,2,6]benzothiadiazocin$11(10 H)$ one 5,5-dioxides, pyrazole, COX inhibitors

\section{Introduction}

Cyclooxygenases (COXs) are key enzymes in the synthesis of prostaglandin $\mathrm{H} 2$ which is a precursor for the biosynthesis of prostaglandins, thromboxanes, and prostacyclins. ${ }^{1} \mathrm{COX}$ enzymes exist in two isoforms, cyclooxygenase-1 (COX-1) and cyclooxygenase-2 (COX-2). $\mathrm{COX}-1$, the constitutive form of the enzyme, is present in the stomach, intestines, kidneys, and platelets. This form is mainly responsible for the physiological production of prostanoids. COX2 , although it is also constitutively expressed in brain, spinal cord, and kidneys, is an inducible form and its expression is triggered under pathological conditions such as inflammation.

In recent years, selective COX-2 inhibitors, such as celecoxib, ${ }^{2}$ rofecoxib, ${ }^{3}$ and valdecoxib ${ }^{4}$ (Figure 1), have been developed as a new generation of non steroidal anti-inflammatory drugs (NSAIDs) with less gastrointestinal and renal toxicity. However, rofecoxib and valdecoxib were withdrawn from the market because of an increased risk of cardiovascular complications (e.g., myocardial infarction and stroke). ${ }^{5,6}$ The high profile NSAID celecoxib (Celebrex; 4-[5-(4methylphenyl)-3-(trifluoromethyl)-1H-pyrazol-1-yl]benzenesulfonamide) contains the pyrazole nucleus. 
Several studies have reported the development of new pyrazole analogues for the development of novel drugs with better safety profiles that could be used long-term to relieve chronic inflammatory conditions. ${ }^{7}$

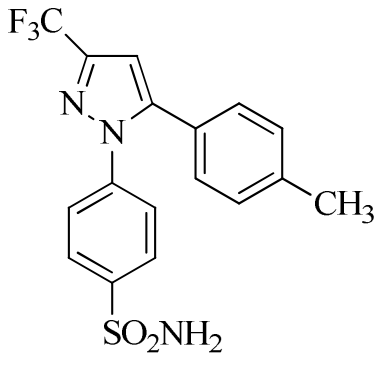

Celecoxib

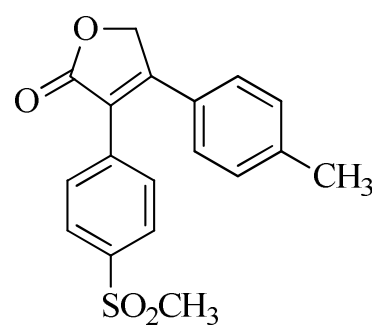

Rofecoxib<smiles>Cc1ccc(-c2c(-c3ccc(S(N)(=O)=O)cc3)noc2C)cc1</smiles>

Valdecoxib

\section{Figure 1}

Recently some of us described the synthesis of pyrazolo[3,4-c][1,5]benzodiazocine derivatives that showed inhibitory profile against both COX1 and COX2. ${ }^{\circ}$

Our starting point was synthesizing new condensed heterocycles containing a pyrazoles moiety in order to investigated how structural changes affect the anti-inflammatory properties. The analgesic and anti-inflammatory activity of substituted dibenzo[ $c, g][1,2,6]$ thiadiazocines has also been reported. ${ }^{9}$

In view of the above facts, and in continuation of our search for anti-inflammatory compounds, we here report the synthesis of new ring system 1,4-dihydropyrazolo[4,3-c][1,2,6] benzothiadiazocin-11(10H)one 5,5-dioxide of type 5 a potential COXs inhibitor.

\section{Results and Discussion}

Pyrazolo-benzothiadiazocines 5a-c were prepared in five or six steps starting from 1-ethyl-3methyl-4-nitro-1H-pyrazole-5-carboxylic acid 1 by esterification with ethereal solution of diazomethane followed by reduction with iron in hydrochloric acid of the corresponding nitromethyl ester, to give the 4-aminopyrazole hydrochloride $\mathbf{2}$ in very good yield. Reaction of compound $\mathbf{2}$ with the 2-nitrobenzensulfonyl chloride, in refluxing toluene, gave the corresponding methyl 1-ethyl-3-methyl-4-\{[(2-nitrophenyl)sulfonyl]amino $\}$-1H-pyrazole-5carboxylate 3a in excellent yield (97\%). The pyrazolyl sulfonamide 3a underwent smooth alkylation at the amide nitrogen, by reaction with methyl or ethyl iodide in warm acetone in the presence of potassium hydroxide, to give the corresponding derivatives $\mathbf{3 b}, \mathbf{c}$ in very good yields (90-95\%).

Reduction of the nitro group of compounds 3a-c with stannous chloride in hydrochloric acid led to the corresponding amines 4a-c in good yields (79-89\%). 
The final cyclization into the tricyclic system was achieved by hydrolysis of the amino derivatives 4a-c in basic medium (ethanol/potassium hydroxide) to give the corresponding carboxylic acids which were not isolated but directly transformed into the 1,4dihydropyrazolo[4,3-c] [1,2,6]benzothiadiazocin-11(10H)one-5,5-dioxide 5a-c by treatment with $\mathrm{SOCl}_{2}$ (Yields 80-89\%). (Scheme 1)

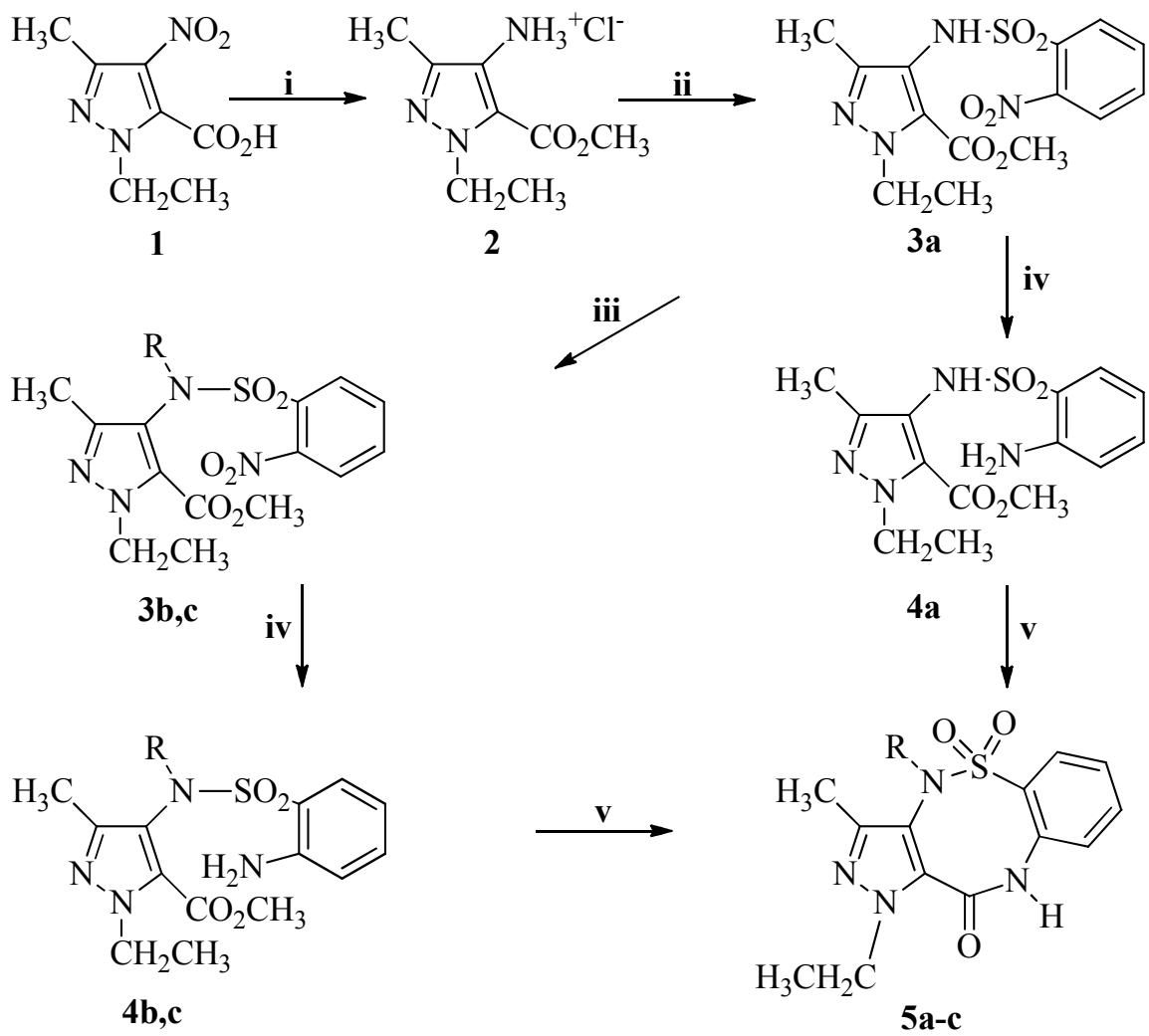

a) $\mathrm{R}=\mathrm{H}$; b) $\mathrm{R}=\mathrm{CH}_{3} ;$ c) $\mathrm{R}=\mathrm{CH}_{2} \mathrm{CH}_{3}$

i: diazomethane/ $\mathrm{Et}_{2} \mathrm{O}$. Yield $100 \%$; then $\mathrm{HCl} / \mathrm{Fe}$, room temperature $(2 \mathrm{~h})$. Yield 93\%; ii: nitrobenzensulfonyl chloride/TEA/Toluene, reflux $(6 \mathrm{~h})$. Yield 97\%; iii: warm acetone/ $\mathrm{KOH}$; RI/Acetone, reflux (20 min). Yields 90-95\%; iv: $\mathrm{HCl} / \mathrm{SnCl}_{2}$, room temperature $(7 \mathrm{~h})$. Yields $79-89 \%$; v: ethanol $/ \mathrm{NaOH} 4 \%$; then $\mathrm{SOCl}_{2}$, reflux (7 h). Yields $80-89 \%$.

Scheme 1. Synthetic route for the preparation of compounds 5a-c.

The structure of the tricyclic derivatives 5a-c was confirmed by spectral data as well as elemental analysis. Thus, in the IR spectra, the NH stretchings were found in the range 3188$3298 \mathrm{~cm}^{-1}$ and the carbonyl absorptions were observed in the range $1645-1687 \mathrm{~cm}^{-1}$. The ${ }^{1} \mathrm{H}$ NMR spectra, beside the benzene protons, showed the methyl group in position 3 as a singlet in the range 2.02-2.12 $\delta$; the pyrazole $\mathrm{N}$-ethyl protons were found at 1.08-1.18 $\delta$ and 4.00-4.04 $\delta$, while the substituent protons next to $\mathrm{N}-4$ nitrogen were observed in the range $3.18-3.65 \delta$; the 
amide hydrogen protons, instead, resonated at 10.09-10.64 $\delta$. In the ${ }^{13} \mathrm{C} N M R$ spectra the carbonyl singlets were observed at 161.6-162.2 ppm.

Compounds 5a, 5b and 5c were tested for their ability to inhibit COX-1 and COX-2 unfortunately none of them showed significant anti-inflammatory activity showing COX-1 inhibition of 5.2, 5.6, and 6.4\% respectively and COX-2 inhibition of 5.3, 5.5, and 6.6\% respectively.

\section{Experimental Section}

General. All melting points were taken on a Büchi-Tottoli capillary apparatus and are uncorrected; IR spectra were determined in bromoform with a SHIMADZU IR Affinity-1 spectrophotometer; ${ }^{1} \mathrm{H}$ and ${ }^{13} \mathrm{C}$ NMR spectra were measured at 200 and $50.3 \mathrm{MHz}$, respectively in DMSO- $d_{6}$ solution, using a Bruker Avance II series $200 \mathrm{MHz}$ spectrometer (TMS as internal reference). Mass spectra were recorded on a JEOL JMS-OI-SG-2 spectrometer at $75 \mathrm{eV}$ (100 $\mu \mathrm{A})$. Elemental analyses $(\mathrm{C}, \mathrm{H}, \mathrm{N})$, performed with a VARIO EL III elemental analyzer, were within $\pm 0.4 \%$ of the theoretical values.

Methyl 4-amino-1-ethyl-3-methyl-1H-pyrazole-5-carboxylate hydrochloride (2) was prepared from the 1-ethyl-3-methyl-4-nitro- $1 H$-pyrazole-5-carboxylic acid ${ }^{10} \mathbf{1}$ which, by reaction with an excess of ethereal solution of diazomethane, was converted into the corresponding methyl ester in quantitative yield, then a solution of the so obtained methyl 1-ethyl-3-methyl-4nitro-1H-pyrazole-5-carboxylate $(2.0 \mathrm{~g}, 9.4 \mathrm{mmol})$ in ethanol $(6 \mathrm{~mL})$, water $(3.5 \mathrm{~mL})$, and hydrochloric acid $(36 \%, 8 \mathrm{~mL})$, powdered iron $(2.0 \mathrm{~g}, 36 \mathrm{mmol})$ was added. The reaction mixture was stirred for 2 hours at room temperature, filtered and washed with warm ethanol. The solution was concentrated under reduced pressure, then, to the obtained residue, acetonitrile (10 $\mathrm{mL})$ and hydrochloric acid $(36 \%, 1 \mathrm{~mL})$ were added. The solid formed was filtered to give derivative 2: white needles, yield 93\%, $1.92 \mathrm{~g}, \mathrm{mp} \mathrm{195-196}{ }^{\circ} \mathrm{C}$; IR $\left(v_{\max }, \mathrm{cm}^{-1}\right): 3227$ and 2854 $\left(\mathrm{NH}_{3}{ }^{+}\right), 1737$ (CO). ${ }^{1} \mathrm{H}$ NMR $\left(200 \mathrm{MHz}, \mathrm{DMSO}-d_{6}\right): \delta_{\mathrm{H}} 1.30\left(3 \mathrm{H}, \mathrm{t}, J=7.1 \mathrm{~Hz}, \mathrm{CCH}_{3}\right), 2.27$ $\left(3 \mathrm{H}, \mathrm{s}, \mathrm{CH}_{3}\right), 3.87\left(3 \mathrm{H}, \mathrm{s}, \mathrm{OCH}_{3}\right), 4.39\left(2 \mathrm{H}, \mathrm{q}, J=7.1 \mathrm{~Hz}, \mathrm{CH}_{2}\right), 9.70\left(3 \mathrm{H}, \mathrm{bs}, \mathrm{NH}_{3}{ }^{+}\right) .{ }^{13} \mathrm{C} \mathrm{NMR}$ $\left(50 \mathrm{MHz}, \mathrm{DMSO}-d_{6}\right): \delta_{\mathrm{C}} 11.0\left(\mathrm{CCH}_{3}\right), 15.4\left(\mathrm{CH}_{3}\right), 46.6\left(\mathrm{CH}_{2}\right), 52.3\left(\mathrm{OCH}_{3}\right), 117.9(\mathrm{C}), 123.9$ (C), 140.2 (C), $158.4(\mathrm{C})$. MS (m/z) $219\left(\mathrm{M}^{+}\right)$; Anal. Calcd for $\mathrm{C}_{8} \mathrm{H}_{14} \mathrm{ClN}_{3} \mathrm{O}_{2}$ : C, 43.74; H, 6.42; N, 19.13\%. Found: C, 43.92; H, 6.54; N, 18.93\%.

\section{Synthesis of methyl 1-ethyl-3-methyl-4-\{[(2-nitrophenyl)sulfonyl]amino $\}-1 H$-pyrazole-5- carboxylate (3a)}

A solution of 4-aminopyrazole hydrochloride 2 (1.09 g, $5 \mathrm{mmol})$, 2-nitrobenzensulfonyl chloride $(2.17 \mathrm{~g}, 5 \mathrm{mmol})$, and triethylamine $(10 \mathrm{mmol})$ in toluene $(100 \mathrm{~mL})$ was heated under reflux for 6 hours. The solvent was then evaporated under reduced pressure and the residue was taken up with water, filtered, and recrystallized from ethanol. 
White needles, yield 97\%, $1.79 \mathrm{~g}, \mathrm{mp} 126-127{ }^{\circ} \mathrm{C}$; IR $\left(\mathrm{v}_{\max }, \mathrm{cm}^{-1}\right): 3558(\mathrm{NH}), 1722(\mathrm{CO}), 1545$ $\left(\mathrm{NO}_{2}\right), 1370$ and $1308\left(\mathrm{SO}_{2}\right) .{ }^{1} \mathrm{H}$ NMR $\left(200 \mathrm{MHz}, \mathrm{DMSO}-d_{6}\right): \delta_{\mathrm{H}} 1.27\left(3 \mathrm{H}, \mathrm{t}, J=7.1 \mathrm{~Hz}, \mathrm{CCH}_{3}\right)$, $2.01\left(3 \mathrm{H}, \mathrm{s}, \mathrm{CH}_{3}\right), 3.35\left(3 \mathrm{H}, \mathrm{s}, \mathrm{OCH}_{3}\right), 4.33\left(2 \mathrm{H}, \mathrm{q}, J=7.1 \mathrm{~Hz}, \mathrm{CH}_{2}\right), 7.73(1 \mathrm{H}, \mathrm{d}, J=7.5 \mathrm{~Hz}, \mathrm{H}-$ 6), $7.80(1 \mathrm{H}, \mathrm{t}, J=7.5 \mathrm{~Hz}, \mathrm{H}-4), 7.88(1 \mathrm{H}, \mathrm{t}, J=7.5 \mathrm{~Hz}, \mathrm{H}-5), 7.97$ (1H, d, $J=7.5 \mathrm{~Hz}, \mathrm{H}-3), 9.74$ $(1 \mathrm{H}, \mathrm{bs}, \mathrm{NH}) .{ }^{13} \mathrm{C}$ NMR $\left(50 \mathrm{MHz}, \mathrm{DMSO}-d_{6}\right): \delta_{\mathrm{C}} 10.8\left(\mathrm{CCH}_{3}\right), 15.5\left(\mathrm{CH}_{3}\right), 46.7\left(\mathrm{CH}_{2}\right), 51.5$ $\left(\mathrm{OCH}_{3}\right), 117.6(\mathrm{C}), 124.1(\mathrm{CH}), 127.7(\mathrm{C}), 130.0(\mathrm{CH}), 132.0(\mathrm{C}), 132.2(\mathrm{CH}), 134.4(\mathrm{CH})$, 145.9 (C), $147.2(\mathrm{C}), 158.7$ (C). MS (m/z) $368\left(\mathrm{M}^{+}\right)$; Anal. Calcd for $\mathrm{C}_{14} \mathrm{H}_{16} \mathrm{~N}_{4} \mathrm{O}_{6} \mathrm{~S}: \mathrm{C}, 45.65 ; \mathrm{H}$, 4.38; N, 15.21\%. Found: C, 45.77; H, 4.15; N, 15.35\%.

General procedure for the synthesis of methyl 1-ethyl-3-methyl-4-\{(R)[(2nitrophenyl)sulfonyl]amino\}-1H-pyrazole-5-carboxylates (3b,c)

To a solution of compound 3a $(1.10 \mathrm{~g}, 3 \mathrm{mmol})$ in warm acetone $(50 \mathrm{~mL})$ powdered potassium hydroxide $(1.0 \mathrm{~g}, 18 \mathrm{mmol})$ was added. The reaction mixture was gently refluxed while alkyl iodide $(7 \mathrm{mmol})$ in anhydrous acetone $(10 \mathrm{~mL})$ was added dropwise. After 20 minutes the solution was concentrated under reduced pressure and the solid formed was washed with water, filtered, and recrystallized from ethanol.

Methyl 1-ethyl-3-methyl-4-\{methyl[(2-nitrophenyl)sulfonyl]amino\}-1H-pyrazole-5carboxylate (3b). $\mathrm{R}=\mathrm{CH}_{3}$. White needles, yield $90 \%, 1.03 \mathrm{~g}, \mathrm{mp} 144-145^{\circ} \mathrm{C} ; \mathrm{IR}\left(\mathrm{v}_{\max }, \mathrm{cm}^{-1}\right)$ : 1723 (CO), $1545\left(\mathrm{NO}_{2}\right), 1371$ and $1308\left(\mathrm{SO}_{2}\right) .{ }^{1} \mathrm{H}$ NMR $\left(200 \mathrm{MHz}, \mathrm{DMSO}-d_{6}\right): \delta_{\mathrm{H}} 1.32(3 \mathrm{H}, \mathrm{t}, J$ $\left.=7.1 \mathrm{~Hz}, \mathrm{CCH}_{3}\right), 2.03\left(3 \mathrm{H}, \mathrm{s}, \mathrm{CH}_{3}\right), 3.28\left(3 \mathrm{H}, \mathrm{s}, \mathrm{NCH}_{3}\right), 3.31\left(3 \mathrm{H}, \mathrm{s}, \mathrm{OCH}_{3}\right), 4.39(2 \mathrm{H}, \mathrm{q}, J=7.1$ $\left.\mathrm{Hz}, \mathrm{CH}_{2}\right), 7.76-8.00$ (4H, m, H-3, H-4, H-5 and H-6). ${ }^{13} \mathrm{C}$ NMR (50 MHz, DMSO- $\left.d_{6}\right): \delta_{\mathrm{C}} 10.7$ $\left(\mathrm{CCH}_{3}\right), 15.4\left(\mathrm{CH}_{3}\right), 39.1\left(\mathrm{NCH}_{3}\right), 47.0\left(\mathrm{CH}_{2}\right), 51.7\left(\mathrm{OCH}_{3}\right), 121.7(\mathrm{C}), 123.9(\mathrm{CH}), 128.4(\mathrm{C})$, $130.4(\mathrm{CH}), 130.9(\mathrm{C}), 132.0(\mathrm{CH}), 134.7(\mathrm{CH}), 146.3(\mathrm{C}), 147.1(\mathrm{C}), 158.3(\mathrm{C}) . \mathrm{MS}(\mathrm{m} / z) 382$ $\left(\mathrm{M}^{+}\right)$; Anal. Calcd for $\mathrm{C}_{15} \mathrm{H}_{18} \mathrm{~N}_{4} \mathrm{O}_{6} \mathrm{~S}: \mathrm{C}, 47.11 ; \mathrm{H}, 4.74 ; \mathrm{N}, 14.65 \%$. Found: $\mathrm{C}, 47.23 ; \mathrm{H}, 4.59 ; \mathrm{N}$, $14.48 \%$.

Methyl 1-ethyl-4\{ ethyl[(2-nitrophenyl)sulfonyl]amino\}-3-methyl-1H-pyrazole-5carboxylate (3c). $\mathrm{R}=\mathrm{CH}_{2}-\mathrm{CH}_{3}$. White needles, yield $95 \%, 1.13 \mathrm{~g}, \mathrm{mp} 105-106{ }^{\circ} \mathrm{C}$; IR $\left(\mathrm{v}_{\max }, \mathrm{cm}^{-}\right.$ $\left.{ }^{1}\right): 1718(\mathrm{CO}), 1545\left(\mathrm{NO}_{2}\right), 1371$ and $1289\left(\mathrm{SO}_{2}\right) .{ }^{1} \mathrm{H}$ NMR $\left(200 \mathrm{MHz}, \mathrm{DMSO}-d_{6}\right): \delta_{\mathrm{H}} 1.09(3 \mathrm{H}$, $\left.\mathrm{t}, J=7.1 \mathrm{~Hz}, \mathrm{CCH}_{3}\right), 1.33\left(3 \mathrm{H}, \mathrm{t}, J=7.1 \mathrm{~Hz}, \mathrm{CCH}_{3}\right), 2.07\left(3 \mathrm{H}, \mathrm{s}, \mathrm{CH}_{3}\right), 3.26\left(3 \mathrm{H}, \mathrm{s}, \mathrm{OCH}_{3}\right), 3.47$ $\left(2 \mathrm{H}, \mathrm{q}, J=7.1 \mathrm{~Hz}, \mathrm{CH}_{2}\right), 3.95\left(2 \mathrm{H}, \mathrm{q}, J=7.1 \mathrm{~Hz}, \mathrm{CH}_{2}\right), 7.74-7.97(4 \mathrm{H}, \mathrm{m}, \mathrm{H}-3, \mathrm{H}-4, \mathrm{H}-5$ and H6). ${ }^{13} \mathrm{C}$ NMR (50 MHz, DMSO- $\left.d_{6}\right): \delta_{\mathrm{C}} 11.0\left(\mathrm{CCH}_{3}\right), 14.5\left(\mathrm{CCH}_{3}\right), 15.4\left(\mathrm{CH}_{3}\right), 47.0\left(\mathrm{CH}_{2}\right), 47.2$ $\left(\mathrm{CH}_{2}\right), 51.5\left(\mathrm{OCH}_{3}\right), 119.3(\mathrm{C}), 123.8(\mathrm{CH}), 128.6(\mathrm{C}), 130.3(\mathrm{CH}), 131.2(\mathrm{C}), 131.9(\mathrm{CH}), 134.6$ $(\mathrm{CH}), 147.1(\mathrm{C}), 147.4(\mathrm{C}), 158.3(\mathrm{C}) . \mathrm{MS}(\mathrm{m} / \mathrm{z}) 396\left(\mathrm{M}^{+}\right)$; Anal. Calcd for $\mathrm{C}_{16} \mathrm{H}_{20} \mathrm{~N}_{4} \mathrm{O}_{6} \mathrm{~S}: \mathrm{C}$, 48.48; H, 5.09; N, 14.13\%. Found: C, 48.62; H, 5.29; N, 14.28\%.

General procedure for the synthesis of methyl 4-\{[(2-aminophenyl)sulfonyl $](R)$ amino $\}-1-$ ethyl-3-methyl-1H-pyrazole-5-carboxylates (4a-c)

Stannous chloride (1.70 g, $9 \mathrm{mmol})$ was dissolved in $\mathrm{HCl}(36 \%, 3 \mathrm{~mL})$ and the resulting solution was cooled to $0{ }^{\circ} \mathrm{C}$. The proper nitroderivative 3a-c $(3 \mathrm{mmol})$ was slowly added and the reaction mixture was kept at room temperature under stirring for 7 hours. Then cold water was added, and 
the solution was neutralized with sodium hydroxide (40\%) and extracted with ethyl acetate. The organic layer was dried over sodium sulfate and after evaporation the obtained residue was purified from small amount of ethanol.

Methyl 4-\{[(2-aminophenyl)sulfonyl]amino\}-1-ethyl-3-methyl-1H-pyrazole-5-carboxylate (4a). R=H. White needles, yield 79\%, $0.80 \mathrm{~g}, \mathrm{mp} 105-106{ }^{\circ} \mathrm{C}$; IR $\left(\mathrm{v}_{\max }, \mathrm{cm}^{-1}\right): 3488(\mathrm{NH}), 3385$ and $3332\left(\mathrm{NH}_{2}\right), 1724(\mathrm{CO}), 1376$ and $1284\left(\mathrm{SO}_{2}\right) .{ }^{1} \mathrm{H}$ NMR $\left(200 \mathrm{MHz}, \mathrm{DMSO}-d_{6}\right): \delta_{\mathrm{H}} 1.21(3 \mathrm{H}$, $\left.\mathrm{t}, J=7.2 \mathrm{~Hz}, \mathrm{CCH}_{3}\right), 1.98\left(3 \mathrm{H}, \mathrm{s}, \mathrm{CH}_{3}\right), 3.56\left(3 \mathrm{H}, \mathrm{s}, \mathrm{OCH}_{3}\right), 4.26\left(2 \mathrm{H}, \mathrm{q}, J=7.2 \mathrm{~Hz}, \mathrm{CH}_{2}\right), 5.91$ $\left(2 \mathrm{H}, \mathrm{bs}, \mathrm{NH}_{2}\right), 6.47\left(1 \mathrm{H}, \mathrm{dt},{ }^{3} J_{H H}=8.0 \mathrm{~Hz},{ }^{4} J_{H H}=1.5 \mathrm{~Hz}, \mathrm{H}-5\right), 6.76\left(1 \mathrm{H}, \mathrm{dd},{ }^{3} J_{H H}=8.0 \mathrm{~Hz},{ }^{4} J_{H H}\right.$ $=1.5 \mathrm{~Hz}, \mathrm{H}-3), 7.09\left(1 \mathrm{H}, \mathrm{dd},{ }^{3} J_{H H}=8.0 \mathrm{~Hz},{ }^{4} J_{H H}=1.5 \mathrm{~Hz}, \mathrm{H}-6\right), 7.22\left(1 \mathrm{H}, \mathrm{dt},{ }^{3} J_{H H}=8.0 \mathrm{~Hz}\right.$, $\left.{ }^{4} J_{H H}=1.5 \mathrm{~Hz}, \mathrm{H}-4\right), 9.13(1 \mathrm{H}, \mathrm{bs}, \mathrm{NH}) .{ }^{13} \mathrm{C} \mathrm{NMR}\left(50 \mathrm{MHz}, \mathrm{DMSO}-d_{6}\right): \delta_{\mathrm{C}} 10.7\left(\mathrm{CCH}_{3}\right), 15.5$ $\left(\mathrm{CH}_{3}\right), 46.4\left(\mathrm{CH}_{2}\right), 51.6\left(\mathrm{OCH}_{3}\right), 114.6(\mathrm{CH}), 116.6(\mathrm{CH}), 118.4(\mathrm{C}), 119.1(\mathrm{C}), 127.8(\mathrm{C}), 128.9$ $(\mathrm{CH}), 133.6(\mathrm{CH}), 145.6(\mathrm{C}), 146.5(\mathrm{C}), 158.9(\mathrm{C}) . \mathrm{MS}(\mathrm{m} / \mathrm{z}) 338\left(\mathrm{M}^{+}\right)$; Anal. Calcd for $\mathrm{C}_{14} \mathrm{H}_{18} \mathrm{~N}_{4} \mathrm{O}_{4} \mathrm{~S}$ : C, 49.69; H, 5.36; N, 16.56\%. Found: C, 49.72; H, 5.26; N, $16.45 \%$.

Methyl 4-\{[(2-aminophenyl)sulfonyl](methyl)amino $\}$-1-ethyl-3-methyl-1H-pyrazole-5carboxylate (4b). $\mathrm{R}=\mathrm{CH}_{3}$. White needles, yield $85 \%, 0.90 \mathrm{~g}, \mathrm{mp} \mathrm{85-86}{ }^{\circ} \mathrm{C}$; IR $\left(v_{\max }, \mathrm{cm}^{-1}\right): 3484$ and $3381\left(\mathrm{NH}_{2}\right), 1719(\mathrm{CO}), 1340$ and $1311\left(\mathrm{SO}_{2}\right) .{ }^{1} \mathrm{H}$ NMR $\left(200 \mathrm{MHz}, \mathrm{DMSO}-d_{6}\right): \delta_{\mathrm{H}} 1.28$ $\left(3 \mathrm{H}, \mathrm{t}, J=7.1 \mathrm{~Hz}, \mathrm{CCH}_{3}\right), 2.01\left(3 \mathrm{H}, \mathrm{s}, \mathrm{CH}_{3}\right), 3.18\left(3 \mathrm{H}, \mathrm{s}, \mathrm{NCH}_{3}\right), 3.49\left(3 \mathrm{H}, \mathrm{s}, \mathrm{OCH}_{3}\right), 4.33(2 \mathrm{H}$, q, $\left.J=7.1 \mathrm{~Hz}, \mathrm{CH}_{2}\right), 5.83\left(2 \mathrm{H}, \mathrm{bs}, \mathrm{NH}_{2}\right), 6.57\left(1 \mathrm{H}, \mathrm{dt},{ }^{3} J_{H H}=8.4 \mathrm{~Hz},{ }^{4} J_{H H}=1.1 \mathrm{~Hz}, \mathrm{H}-5\right), 6.82$ $\left(1 \mathrm{H}, \mathrm{dd},{ }^{3} J_{H H}=8.4 \mathrm{~Hz},{ }^{4} J_{H H}=1.1 \mathrm{~Hz}, \mathrm{H}-3\right), 7.23-7.30\left(2 \mathrm{H}, \mathrm{m}, \mathrm{H}-4\right.$ and H-6). ${ }^{13} \mathrm{C}$ NMR $(50$ $\left.\mathrm{MHz}, \mathrm{DMSO}-d_{6}\right): \delta_{\mathrm{C}} 10.8\left(\mathrm{CCH}_{3}\right), 15.5\left(\mathrm{CH}_{3}\right), 38.4\left(\mathrm{NCH}_{3}\right), 46.7\left(\mathrm{CH}_{2}\right), 51.7\left(\mathrm{OCH}_{3}\right), 115.1$ $(\mathrm{CH}), 117.1(\mathrm{CH}), 119.3(\mathrm{C}), 123.0(\mathrm{C}), 128.9(\mathrm{C}), 129.4(\mathrm{CH}), 133.8(\mathrm{CH}), 146.3(\mathrm{C}), 146.9$ (C), $158.8(\mathrm{C})$. MS (m/z) $352\left(\mathrm{M}^{+}\right)$; Anal. Calcd for $\mathrm{C}_{15} \mathrm{H}_{20} \mathrm{~N}_{4} \mathrm{O}_{4} \mathrm{~S}$ : C, 51.12; H, 5.72; N, 15.90\%. Found: C, 51.33; H, 5.56; N, 15.64\%.

Methyl 4-\{[(2-aminophenyl)sulfonyl](ethyl)amino\}-1-ethyl-3-methyl-1H-pyrazole-5carboxylate (4c). $\mathrm{R}=\mathrm{CH}_{2}-\mathrm{CH}_{3}$. White needles, yield 89\%, $0.98 \mathrm{~g}, \mathrm{mp} 76-77{ }^{\circ} \mathrm{C}$; IR $\left(\mathrm{v}_{\max }, \mathrm{cm}^{-1}\right)$ : 3483 and $3381\left(\mathrm{NH}_{2}\right), 1719(\mathrm{CO}), 1339$ and $1289\left(\mathrm{SO}_{2}\right) .{ }^{1} \mathrm{H}$ NMR $\left(200 \mathrm{MHz}, \mathrm{DMSO}-d_{6}\right): \delta_{\mathrm{H}}$ $1.05\left(3 \mathrm{H}, \mathrm{t}, J=7.0 \mathrm{~Hz}, \mathrm{CCH}_{3}\right), 1.28\left(3 \mathrm{H}, \mathrm{t}, J=7.0 \mathrm{~Hz}, \mathrm{CCH}_{3}\right), 2.02\left(3 \mathrm{H}, \mathrm{s}, \mathrm{CH}_{3}\right), 3.45(3 \mathrm{H}, \mathrm{s}$, $\left.\mathrm{OCH}_{3}\right), 3.78\left(2 \mathrm{H}, \mathrm{q}, J=7.0 \mathrm{~Hz}, \mathrm{CH}_{2}\right), 4.34\left(2 \mathrm{H}, \mathrm{q}, J=7.0 \mathrm{~Hz}, \mathrm{CH}_{2}\right), 5.80\left(2 \mathrm{H}, \mathrm{bs}, \mathrm{NH}_{2}\right), 6.55$ $(1 \mathrm{H}, \mathrm{t}, J=8.4 \mathrm{~Hz}, \mathrm{H}-5), 6.80(1 \mathrm{H}, \mathrm{d}, J=8.4 \mathrm{~Hz}, \mathrm{H}-3), 7.24-7.28(2 \mathrm{H}, \mathrm{m}, \mathrm{H}-4$ and $\mathrm{H}-6) .{ }^{13} \mathrm{C}$ NMR (50 MHz, DMSO- $\left.d_{6}\right): \delta_{\mathrm{C}} 10.9\left(\mathrm{CCH}_{3}\right), 14.5\left(\mathrm{CCH}_{3}\right), 15.4\left(\mathrm{CH}_{3}\right), 45.9\left(\mathrm{CH}_{2}\right), 46.9\left(\mathrm{CH}_{2}\right)$, $51.6\left(\mathrm{OCH}_{3}\right), 115.1(\mathrm{CH}), 117.1(\mathrm{CH}), 119.9(\mathrm{C}), 120.6(\mathrm{C}), 129.2(\mathrm{C}), 129.3(\mathrm{CH}), 133.7(\mathrm{CH})$, 146.7 (C), 147.4 (C), 158.7 (C). MS (m/z) $366\left(\mathrm{M}^{+}\right)$; Anal. Calcd for $\mathrm{C}_{16} \mathrm{H}_{22} \mathrm{~N}_{4} \mathrm{O}_{4} \mathrm{~S}: \mathrm{C}, 52.44 ; \mathrm{H}$, 6.05; N, 15.29\%. Found: C, 52.33; H, 6.26; N, 15.34\%.

General procedure for the synthesis of 1-ethyl-3-methyl-4- $R$-1,4-dihydropyrazolo[4,3c][1,2,6]benzothiadiazocin-11(10H)-one 5,5-dioxides (5a-c)

A solution of the proper amine 4 a-c $(3 \mathrm{mmol})$ in ethanol $(15 \mathrm{~mL})$ and aqueous sodium hydroxide $(4 \%, 30 \mathrm{~mL})$ was refluxed for 20 minutes. The reaction mixture was concentrate and acidified to pH 5.0 with $\mathrm{NH}_{4} \mathrm{Cl}$. The solid formed was collected and dried. The crude residue was treated 
with $\mathrm{SOCl}_{2}(20 \mathrm{~mL})$ and gently heated for 7 hours. Upon evaporation the residue was washed with water, filtered and recrystallized from ethanol.

1-Ethyl-3-methyl-1,4-dihydropyrazolo[4,3-c][1,2,6]benzothiadiazocin-11(10H)-one $\quad 5,5-$ dioxide (5a). $\mathrm{R}=\mathrm{H}$. White needles, yield $80 \%, 0.75 \mathrm{~g}, \mathrm{mp} 228-229{ }^{\circ} \mathrm{C}$; IR $\left(\mathrm{v}_{\max }, \mathrm{cm}^{-1}\right): 3570$ $(\mathrm{NH}), 3346(\mathrm{NH}), 1677(\mathrm{CO}), 1318$ and $1297\left(\mathrm{SO}_{2}\right) .{ }^{1} \mathrm{H}$ NMR $\left(200 \mathrm{MHz}, \mathrm{DMSO}-d_{6}\right): \delta_{\mathrm{H}} 1.18$ $\left(3 \mathrm{H}, \mathrm{t}, J=7.1 \mathrm{~Hz}, \mathrm{CCH}_{3}\right), 2.02\left(3 \mathrm{H}, \mathrm{s}, \mathrm{CH}_{3}\right), 4.04\left(2 \mathrm{H}, \mathrm{q}, J=7.1 \mathrm{~Hz}, \mathrm{CH}_{2}\right), 7.28\left(1 \mathrm{H}, \mathrm{dd},{ }^{3} J_{H H}=\right.$ $\left.7.9 \mathrm{~Hz},{ }^{4} J_{H H}=1.6 \mathrm{~Hz}, \mathrm{H}-9\right), 7.53\left(1 \mathrm{H}, \mathrm{dt},{ }^{3} J_{H H}=7.9 \mathrm{~Hz},{ }^{4} J_{H H}=1.6 \mathrm{~Hz}, \mathrm{H}-7\right), 7.69\left(1 \mathrm{H}, \mathrm{dt},{ }^{3} J_{H H}\right.$ $\left.=7.9 \mathrm{~Hz},{ }^{4} J_{H H}=1.6 \mathrm{~Hz}, \mathrm{H}-8\right), 7.91\left(1 \mathrm{H}, \mathrm{dd},{ }^{3} J_{H H}=7.9 \mathrm{~Hz},{ }^{4} J_{H H}=1.6 \mathrm{~Hz}, \mathrm{H}-6\right), 10.09(1 \mathrm{H}, \mathrm{bs}$, $\mathrm{NH}), 10.43(1 \mathrm{H}, \mathrm{bs}, \mathrm{NH}) .{ }^{13} \mathrm{C}$ NMR $\left(50 \mathrm{MHz}, \mathrm{DMSO}-d_{6}\right): \delta_{\mathrm{C}} 10.9\left(\mathrm{CCH}_{3}\right), 15.7\left(\mathrm{CH}_{3}\right), 45.4$ $\left(\mathrm{CH}_{2}\right), 116.7(\mathrm{C}), 125.3(\mathrm{C}), 127.6(\mathrm{CH}), 128.0(\mathrm{CH}), 128.7(\mathrm{CH}), 131.7(\mathrm{C}), 134.4(\mathrm{CH}), 135.4$ (C), $136.1(\mathrm{C}), 161.6(\mathrm{C})$. MS (m/z) $306\left(\mathrm{M}^{+}\right)$; Anal. Calcd for $\mathrm{C}_{13} \mathrm{H}_{14} \mathrm{~N}_{4} \mathrm{O}_{3} \mathrm{~S}$ : C, 50.97; H, 4.61; N, 18.29\%. Found: C, 50.75; H, 4.83; N, $18.35 \%$.

1-Ethyl-3,4-dimethyl-1,4-dihydropyrazolo[4,3-c][1,2,6]benzothiadiazocin-11(10H)one 5,5dioxide (5b). $\mathrm{R}=\mathrm{CH}_{3}$. White needles, yield 86\%, 0.83 g, mp 190-191 ${ }^{\circ} \mathrm{C}$; IR $\left(\mathrm{v}_{\max }, \mathrm{cm}^{-1}\right): 3340$ $(\mathrm{NH}), 1681$ (CO), 1320 and $1299\left(\mathrm{SO}_{2}\right) .{ }^{1} \mathrm{H}$ NMR $\left(200 \mathrm{MHz}, \mathrm{DMSO}-d_{6}\right): \delta_{\mathrm{H}} 1.12(3 \mathrm{H}, \mathrm{t}, J=7.2$ $\left.\mathrm{Hz}, \mathrm{CCH}_{3}\right), 2.10\left(3 \mathrm{H}, \mathrm{s}, \mathrm{CH}_{3}\right), 3.18\left(3 \mathrm{H}, \mathrm{s}, \mathrm{NCH}_{3}\right), 4.00\left(2 \mathrm{H}, \mathrm{q}, J=7.2 \mathrm{~Hz}, \mathrm{CH}_{2}\right), 7.34(1 \mathrm{H}$, dd, $\left.{ }^{3} J_{H H}=8.0 \mathrm{~Hz},{ }^{4} J_{H H}=1.5 \mathrm{~Hz}, \mathrm{H}-9\right), 7.49\left(1 \mathrm{H}, \mathrm{dt},{ }^{3} J_{H H}=8.0 \mathrm{~Hz},{ }^{4} J_{H H}=1.5 \mathrm{~Hz}, \mathrm{H}-7\right), 7.68(1 \mathrm{H}$, $\left.\mathrm{dt},{ }^{3} J_{H H}=8.0 \mathrm{~Hz},{ }^{4} J_{H H}=1.5 \mathrm{~Hz}, \mathrm{H}-8\right), 7.94\left(1 \mathrm{H}, \mathrm{dd},{ }^{3} J_{H H}=8.0 \mathrm{~Hz},{ }^{4} J_{H H}=1.5 \mathrm{~Hz}, \mathrm{H}-6\right), 10.64$ $(1 \mathrm{H}, \mathrm{bs}, \mathrm{NH}) .{ }^{13} \mathrm{C} \mathrm{NMR}\left(50 \mathrm{MHz}, \mathrm{DMSO}-d_{6}\right): \delta_{\mathrm{C}} 10.9\left(\mathrm{CCH}_{3}\right), 15.4\left(\mathrm{CH}_{3}\right), 36.7\left(\mathrm{NCH}_{3}\right), 45.3$ $\left(\mathrm{CH}_{2}\right), 121.2(\mathrm{C}), 128.3(\mathrm{CH}), 128.5(\mathrm{CH}), 130.2(\mathrm{CH}), 130.6(\mathrm{C}), 132.8(\mathrm{C}), 134.6(\mathrm{CH}), 135.3$ (C), $142.4(\mathrm{C}), 161.9(\mathrm{C})$. MS (m/z) $320\left(\mathrm{M}^{+}\right)$; Anal. Calcd for $\mathrm{C}_{14} \mathrm{H}_{16} \mathrm{~N}_{4} \mathrm{O}_{3} \mathrm{~S}$ : C, 52.49; H, 5.03; N, 17.49\%. Found: C, 52.37; H, 5.16; N, 17.35\%.

1,4-Diethyl-3-methyl-1,4-dihydropyrazolo[4,3-c][1,2,6]benzothiadiazocin-11(10H)-one $5,5-$ dioxide (5c). $\mathrm{R}=\mathrm{CH}_{2}-\mathrm{CH}_{3}$. White needles, yield $89 \%$, mp 194-195 ${ }^{\circ} \mathrm{C}$; IR $\left(\mathrm{v}_{\max }, \mathrm{cm}^{-1}\right): 3343$ $(\mathrm{NH}), 1683(\mathrm{CO}), 1327$ and $1301\left(\mathrm{SO}_{2}\right) .{ }^{1} \mathrm{H}$ NMR $\left(200 \mathrm{MHz}, \mathrm{DMSO}-d_{6}\right): \delta_{\mathrm{H}} 1.08(3 \mathrm{H}, \mathrm{t}, J=7.1$ $\left.\mathrm{Hz}, \mathrm{CCH}_{3}\right), 1.10\left(3 \mathrm{H}, \mathrm{t}, J=7.1 \mathrm{~Hz}, \mathrm{CCH}_{3}\right), 2.12\left(3 \mathrm{H}, \mathrm{s}, \mathrm{CH}_{3}\right), 3.65\left(2 \mathrm{H}, \mathrm{q}, J=7.1 \mathrm{~Hz}, \mathrm{CH}_{2}\right)$, $4.02\left(2 \mathrm{H}, \mathrm{q}, J=7.1 \mathrm{~Hz}, \mathrm{CH}_{2}\right), 7.34\left(1 \mathrm{H}, \mathrm{dd},{ }^{3} J_{H H}=7.9 \mathrm{~Hz},{ }^{4} J_{H H}=1.3 \mathrm{~Hz}, \mathrm{H}-9\right), 7.47(1 \mathrm{H}, \mathrm{dt}$, $\left.{ }^{3} J_{H H}=7.9 \mathrm{~Hz},{ }^{4} J_{H H}=1.3 \mathrm{~Hz}, \mathrm{H}-7\right), 7.65\left(1 \mathrm{H}, \mathrm{dt},{ }^{3} J_{H H}=7.9 \mathrm{~Hz},{ }^{4} J_{H H}=1.3 \mathrm{~Hz}, \mathrm{H}-8\right), 7.94(1 \mathrm{H}$, $\left.\mathrm{dd},{ }^{3} J_{H H}=7.9 \mathrm{~Hz},{ }^{4} J_{H H}=1.3 \mathrm{~Hz}, \mathrm{H}-6\right), 10.53(1 \mathrm{H}, \mathrm{bs}, \mathrm{NH}) .{ }^{13} \mathrm{C}$ NMR $\left(50 \mathrm{MHz}, \mathrm{DMSO}-d_{6}\right): \delta_{\mathrm{C}}$ $10.5\left(\mathrm{CCH}_{3}\right), 14.2\left(\mathrm{CCH}_{3}\right), 15.5\left(\mathrm{CH}_{3}\right), 45.1\left(\mathrm{CH}_{2}\right), 45.2\left(\mathrm{CH}_{2}\right), 118.9(\mathrm{C}), 128.5(\mathrm{CH}), 129.1$ (CH), $130.2(\mathrm{CH}), 132.7(\mathrm{C}), 134.1(\mathrm{CH}), 134.4(\mathrm{C}), 134.6(\mathrm{C}), 144.4(\mathrm{C}), 162.2(\mathrm{C}) . \mathrm{MS}(\mathrm{m} / \mathrm{z})$ $334\left(\mathrm{M}^{+}\right)$; Anal. Calcd for $\mathrm{C}_{15} \mathrm{H}_{18} \mathrm{~N}_{4} \mathrm{O}_{3} \mathrm{~S}$ : C, 53.88; H, 5.43; N, 16.75\%. Found: C, 53.92; H, 5.35; $\mathrm{N}, 16.66 \%$.

\section{Biology}

In vitro cyclooxygenase inhibition assay. The reference compounds (indomethacin and NS398) were purchased from Cayman Chemical, Ann Arbor, MI (cat. N. 70270 and 70590 respectively). They are used at the final concentration of $0.2 \mu \mathrm{M}$, according to the manufacturer's instructions. Compounds 5a-c were tested for their ability to inhibit COX-1 and COX-2 using a COX-(ovine) inhibitor screening kit (Catalogue No 560101, Cayman Chemical, Ann Arbor, MI) according to 
the manufacturer's instructions. Cyclooxygenase catalyzes the first step in the biosynthesis of arachidonic acid (AA) to PGH2 PGF2 $\alpha$ produced from PGH2 by reduction with stannous chloride is measured by enzyme immunoassay (ACE competitive EIA). Stock solutions of test compounds were dissolved in a minimum volume of DMSO. Briefly, to a series of supplied reaction buffer solutions $(950 \mu \mathrm{L}, 0.1 \mathrm{M}$ Tris-HCI pH 8.0 containing $5 \mathrm{mM}$ EDTA and $2 \mathrm{mM}$ phenol) with either COX 1 or COX-2 $(10 \mu \mathrm{L})$ enzyme in the presence of heme $(10 \mu \mathrm{L})$ was added $20 \mu \mathrm{L}$ of $10 \mu \mathrm{M}$ concentration of test drug solutions. These solution were incubated for a period of $2 \mathrm{~min}$ at $37{ }^{\circ} \mathrm{C}$ after which $10 \mu \mathrm{L}$ of AA $(100 \mu \mathrm{M})$ was added, and the COX reaction was stopped by the addition of $50 \mu \mathrm{L}$ of $1 \mathrm{M}$ HCI after 2 min. PGF2 produced from PGH2 by reduction with stannous chloride, was measured by enzyme immunoassay. This assay is based on the competition between PGs and a PG-acetylcholinesterase conjugate (PG tracer) for a limited amount of PG antiserum. The amount of PG tracer that is able to bind to the PG antiserum is inversely proportional to the concentration of PGs in the wells, since the concentration of the PG tracer is held constant while the concentration of PGs varies. This antibody-PG complex binds to a mouse anti-rabbit monoclonal antibody that had been attached to the well previously. The plate is washed to remove any unbound reagents and then Ellman's Reagent, which contains the substrate to acetylcholinesterase is added to the well. The product of this enzymatic reaction produces a distinct yellow color that absorbs at $405 \mathrm{~nm}$. The intensity of this color, determined spectrophotometrically, is proportional to the amount of PG tracer bound to the well, which is inversely proportional to the amount of PGs present in the well during the incubation absorbance $\propto$ [bound PG tracer] $\propto 1 /$ PGs. Percent inhibition was calculated by comparison of compoundtreated to various control incubations (duplicate determinations).

\section{Acknowledgements}

This work was financially supported by Ministero dell'Istruzione dell'Università e della Ricerca

\section{References}

1. (a) Vane, J. R. Nat. New Biol. 1971, 231, 232. (b) Dannhardt, G.; Laufer, S. Curr. Med. Chem. 2000, 7, 1101.

2. Penning, T. D.; Talley, J. J.; Bertenshaw, S. R.; Carter, J. S.; Collins, P. W.; Docter, S.; Graneto, M. J.; Lee, L. F.; Malecha, J. W.; Miyashiro, J. M.; Rogers, R. S.; Rogier, D. J.; Yu, S. S.; Anderson, G. D.; Burton, E. G.; Cogburn, J. N.; Gregory, S. A.; Koboldt, C. M.; Perkins, W. E.; Seibert, K.; Veenhuizen, A. W.; Zhang, Y. Y.; Isakson, P. C. J. Med. Chem. 1997, 40, 1347.

3. Prasit, P.; Wang, Z.; Brideau, C.; Chan, C. C.; Charleson, S.; Cromlish, W.; Ethier, D.; Evans, J. F.; Ford-Hutchinson, A. W.; Gauthier, J. Y.; Gordon, R.; Guay, J.; Gresser, M.; 
Kargman, S.; Kennedy, B.; Leblanc, Y.; Leger, S.; Mancini, J.; O’Neill, G. P.; Ouellet, M.; Percival, M. D.; Perrier, H.; Riendeau, D.; Rodger, I.; Zamboni, R. Bioorg. Med. Chem. Lett. 1999, 9, 1773.

4. Talley, J. J.; Brown, D. L.; Carter, J. S.; Graneto, M. J.; Koboldt, C. M.; Masferrer, J. L.; Perkins, W. E.; Rogers, R. S.; Shaffer, A. F.; Zhang, Y. Y.; Zweifel, B. S.; Seibert, K. J. Med. Chem. 2000, 43, 775.

5. Dogne, J. M.; Supuran, C. T.; Pratico, D. J. Med. Chem. 2005, 48, 2251.

6. Solomon, D. H. Arthritis Rheum. 2005, 52, 1968.

7. (a) Dressen, D.; Garofalo, A. W.; Hawkinson, J.; Hom, D.; Jagodzinski, J.; Marugg, J. L.; Neitzel, M. L.; Pless, M. A.; Szoke, B.; Tung, J. S.; Wone, D. W. G.; Wu, J.; Zhang, H. J. Med. Chem. 2007, 50, 5161. (b) Navidpour, L., Shadnia, H.; Shafaroodi, H.; Amini, M.; Dehupor, A. R.; Shafiee, A. Bioorg. Med. Chem. 2007, 15, 1976; (c) Ranatunge, R. R.; Augustynlak, M.; Bandarange, U. K.; Earl, R. A., Ellis, J. L.; Garvey, D. S.; Janero, D. R.; Gordon Letts, L.; Martino, A. M.; Murty, M. G.; Richardson, S. T.; Schroeder, J. D.; Shumway, M. J.; Tam, S. W.; Trocha, A. M.; Young, D. V. J. Med. Chem. 2004, 47, 2180.

8. Migliara, O.; Raffa, D.; Plescia, S.; Cusimano, M. G.; Carbone, A. Arkivoc 2009, (ii), 1.

9. Nakanishi, M.; Munakata, T.; Tsumagari, T. Ger. Offen. (1970), DE1913920; Chem. Abstr. 1970, 74, 22908.

10. DeWald, H. A.; Nordin, I. C.; L'Italien, Y. J.; Parcell, R. F. J. Med. Chem. 1973, 16, 1346. 